University of Nebraska - Lincoln

DigitalCommons@University of Nebraska - Lincoln

Other Publications in Zoonotics and Wildlife

Disease

Wildlife Disease and Zoonotics

1995

\title{
Guidelines for Working with Rodents Potentially Infected with Hantavirus
}

James N. Mills

Centers for Disease Control and Prevention

Terry L. Yates

University of New Mexico

James E. Childs

Centers for Disease Control and Prevention, james.childs@yale.edu

Robert R. Parmenter

University of New Mexico

Thomas G. Ksiazek

Centers for Disease Control and Prevention

See next page for additional authors

Follow this and additional works at: https://digitalcommons.unl.edu/zoonoticspub

Part of the Veterinary Infectious Diseases Commons

Mills, James N.; Yates, Terry L.; Childs, James E.; Parmenter, Robert R.; Ksiazek, Thomas G.; Rollin, Pierre E.; and Peters, C.J., "Guidelines for Working with Rodents Potentially Infected with Hantavirus" (1995). Other Publications in Zoonotics and Wildlife Disease. 84.

https://digitalcommons.unl.edu/zoonoticspub/84

This Article is brought to you for free and open access by the Wildlife Disease and Zoonotics at DigitalCommons@University of Nebraska - Lincoln. It has been accepted for inclusion in Other Publications in Zoonotics and Wildlife Disease by an authorized administrator of DigitalCommons@University of Nebraska - Lincoln. 


\section{Authors}

James N. Mills, Terry L. Yates, James E. Childs, Robert R. Parmenter, Thomas G. Ksiazek, Pierre E. Rollin, and C.J. Peters 


\title{
GUIDELINES FOR WORKING WITH RODENTS POTENTIALLY INFECTED WITH HANTAVIRUS
}

\author{
James N. Mills, Terry L. Yates, James E. Childs, Robert R. Parmenter, \\ Thomas G. KsiazeK, Pierre E. Rollin, and C. J. Peters \\ Viral and Rickettsial Zoonoses Branch, National Center for Infectious Diseases, \\ Centers for Disease Control and Prevention, 1600 Clifton Road NE, \\ Atlanta, GA 30333 (JNM, JEC, TGK, PER, CJP) \\ Department of Biology and Museum of Southwestern Biology, \\ University of New Mexico, Albuquerque, NM 87131 (TLY, RRP)
}

Because of the high morbidity and mortality associated with hantavirus pulmonary syndrome and the possibility of aerosol transmission of hantaviruses, persons handling known reservoir species in the field, laboratory, or classroom should take special precautions to minimize the risk of infection. We provide specific guidelines for personal safety while trapping, handling and releasing, transporting, sampling, and performing necropsy on potentially infected rodents or teaching field classes in areas occupied by reservoir species. Special consideration should be given to respiratory protection, choice and use of disinfectants, decontamination of instruments and traps, proper disposal of infectious wastes, and preservation and shipment of samples intended for hantavirus testing. Precautionary testing of wild rodents used to start laboratory colonies is recommended. Although we specifically address hantaviruses, the procedures described are applicable for any study of populations of small mammals when an infectious zoonotic agent transmissible by aerosol and capable of causing high morbidity and mortality is involved.

Key words: Hantavirus, laboratory colonies, hantavirus pulmonary syndrome, safety, necropsy, Peromyscus, Sigmodon

Hantavirus pulmonary syndrome (HPS) was recognized in 1993 as an acute disease caused by several related strains of viruses in the genus Hantavirus, family Bunyaviridae. The deer mouse, Peromyscus maniculatus, is the primary reservoir for Sin Nombre virus (SNV) and related strains of hantavirus throughout much of North America. It is possible that other species of Peromyscus serve as competent hosts, and a variety of species have been shown to have antibody (Childs et al., 1994). Black Creek Canal virus (BCCV), isolated from Sigmodon hispidus, was responsible for at least one case of HPS in Florida (Rollin et al., in press). Nucleotide sequences suggestive of novel species of Hantavirus have been identified by polymerase chain reaction from Reithrodontomys megalotis (Hjelle et al., 1994) and Microtus californicus
(B. Hjelle, pers. comm.), but no relationship to human disease has been demonstrated. It is likely that hantaviruses will be found in other species of rodents and their potential for pathogenicity in humans will remain in question except in cases in which a clear relationship to human disease is found. Prior to 1993, two hantaviruses were known to exist in the United States. Seoul virus, from Rattus norvegicus, causes mild hemorrhagic fever with renal syndrome in Asia, but rarely is associated with human disease in the United States (Glass et al., 1993). Prospect Hill virus, from Microtus pennsylvanicus (Lee et al., 1985), has not been associated with human disease. For a more complete list of reservoir species of hantavirus, geographic distributions, and associated diseases, see Childs et al. (1995:table $1)$. 
Rodent hosts that are infected with hantavirus may develop a life-long chronic infection and shed infectious virus in urine, feces, and saliva (LeDuc, 1987). The primary mode of infection to humans is thought to be inhalation of aerosolized virus, although rodent bites and direct contact with broken skin or mucous membranes also are potential sources of infection (Tsai, 1987).

With the appearance of HPS, researchers, health officials, and university instructors who routinely work with wild rodents have realized that they are potentially at risk of infection with this newly discovered virus. The risk of exposure varies with professional activities. People who may have a relatively high risk of exposure to infected rodents include mammalogists and public health workers, who may be sampling infected rodents, especially in areas of recent disease activity. Field researchers conducting mark-recapture studies on rodents or students and instructors in classes on field trips to collect or observe rodents also may be subject to exposure to the virus.

Because of the high morbidity and mortality associated with HPS and the likelihood of aerosol transmission of virus, persons handling known reservoir species in the field or laboratory should take special precautions to minimize the risk of infection. The recommendations provided in this article constitute a guide for those persons performing field and laboratory studies involving populations of rodents that potentially are infected with hantavirus. Howev$\mathrm{er}$, the procedures outlined are appropriate for any study of populations of small mammals when an infectious zoonotic agent that can cause high morbidity and mortality is involved. Previous general recommendations for risk reduction have been published (Centers for Disease Control and Prevention, 1993).

Persons contemplating trapping, handling, or performing necropsies on rodents in areas of hantavirus transmission should contact their state public health agency for local recommendations that may complement these guidelines. Local training, including an orientation about hantavirus transmission and specific safety procedures, is recommended. General background information concerning hantaviruses and hantavirus disease may be found in other sources (Childs et al., 1994, 1995; Duchin et al., 1994; LeDuc, 1987; McKee et al., 1991; Tsai, 1987).

\section{FiELD METHODS}

Setting rodent traps.-Live-capture traps, such as Sherman or Tomahawk traps, are preferred for obtaining specimens when blood samples, tissues, or chromosome preparations are required. Traps that have contained captured rodents should be disinfected prior to placement in the field (see trap-cleaning guidelines under "cleanup"). No special clothing or equipment is required for setting and baiting clean traps, although a long-sleeved shirt, long pants, socks, and lace-up shoes are recommended for all fieldwork.

Retrieving trapped rodents.-Workers checking and retrieving traps should wear thick rubber gloves in addition to the protective clothing recommended. Coveralls that can be removed when trapping and processing are completed may provide an additional measure of safety. When a closed trap is encountered, the worker should lift it without shaking it and, standing with the wind to the left or right side and the trap held at arm's length, push the door open just enough to peer inside. Traps containing rodents should be placed in double plastic bags and transported as quickly as possible to the processing site, keeping the animals out of direct sunlight to protect them from overheating. Gloved hands should be washed with soap and water or a suitable disinfectant (e.g., 1\% household bleach, 5\% hospital-type bulk Lysol; National Laboratories, Lehn and Fink Industrial Products Division, Montvale, NJ) or any hospital-grade disinfectant approved by the Environmental Protection Agency used according to the manufacturer's instructions. As a surface disinfectant, 1\% household bleach is adequate, but $10 \%$ bleach is more effective for heavily soiled items or areas contaminated with feces or nesting materials of rodents. Hands should be thoroughly washed with soap and water immediately after removing 


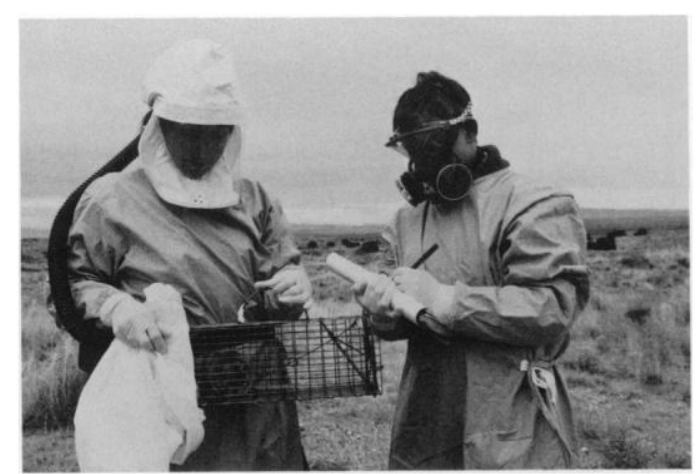

FIG. 1.-Technicians from the University of New Mexico, Museum of Southwestern Biology prepare to mark and release a white-throated woodrat (Neotoma albigula) at the Sevilleta Long Term Ecological Research site in New Mexico. The technician on the left is wearing a full-hood powered air-purifying respirator with high efficiency particulate air (HEPA) filters; the technician on the right is wearing goggles and a half-face respirator with HEPA filters. Note disposable gowns, gloves, and plastic bags for transporting the rodent and trap.

gloves. Bagged traps should be transported in the back of a pickup truck or other compartment isolated from passenger sections.

Handling live rodents.-The field processing site should be in a secluded area, away from other humans or domestic animals. Table and other work surfaces, chairs, and floor should be of a nonporous material that can be easily disinfected and cleaned. Weather permitting, outdoor processing is preferred because of greater ventilation and the antiviral effects of natural ultraviolet light. Workers should sit with the wind from behind at an ca. $45^{\circ}$ angle; captured animals should be located downwind, and vehicles and equipment should be upwind. If indoors, there should be adequate means of ventilating the area to the outside. Workers should wear protective clothing, including a surgeon's gown or coveralls (preferably disposable), shoe covers, latex gloves (two pairs), suitable eye protection, and a respirator. Respirators may be of the negative-pressure type or a powered air-purifying respirator. Either type should be fitted with high-efficiency particulate air (HEPA) filters (Fig. 1). Establishment of a comprehensive respiratory protection program, including proper fitting, pulmonary function test, and instruction on respirator care and use is required before using any respirator (Ref. 29CFR1910.134, OSHA Respiratory Protection Standard). Necessary guidance can be provided by the local health department or the Area Office of the Occupational Health and Safety Administration. The safety office of most colleges and universities will provide specific guidelines and procedures for obtaining and using respirators.

If animals are not anesthetized (as in the case of demonstrations or observations during university field trips, or during certain mark-release studies on populations of rodents), gloves of sufficient thickness to prevent rodent bites should be worn by handlers. Protective gloves, goggles, and respirators also should be worn when releasing live animals from traps. Following release of animals, traps should be transported in double plastic bags and decontaminated as subsequently described. Soiled trap bedding and any remaining bait or feces should be treated as biohazardous waste and incinerated or autoclaved prior to disposal.

Collection of blood samples.-Animals in live-capture traps may be anesthetized by placing the entire trap into a plastic bag containing gauze or cotton soaked with an inhalant anesthetic. Alternatively, animals in Sherman traps may be shaken into a clear plastic bag containing the anesthetic. Technicians should take care to minimize inhaling anesthetic during these procedures. Because of the hepatotoxicity of halothane and chloroform and the flammability of ether, we recommend the use of methoxyflurane (Metofane) as the primary anesthetic. Blood samples should be obtained from the retro-orbital sinus of profoundly anesthetized animals by using a heparinized capillary tube or Pasteur pipette. Blood can be allowed to drip from the capillary tube into a 1-2-ml plastic cryovial (e.g., Nunc, Sarstedt, Evergreen). At least four or five drops of blood are required for laboratory testing for hantaviruses. Use of needles for cardiac puncture should be avoided because of the risk of needle-stick injury and infection. The capillary tube or pipette should be discarded in a disposable "sharps" container. The bleeding then should be stopped by pinching the rodent's eye closed with a gauze square and applying pressure to the orbit. Gloves, working surfaces, and outside of the vial should be cleaned, if necessary, using a suitable disinfectant (as previously described) and paper towels. The anesthe- 
tized animal can be euthanized by cervical dislocation or by overdose of anesthetic before necropsy. In areas of plague endemicity (west of the 101 st meridian), fleas can be killed by placing the animal into a tightly sealed container with cotton or gauze wetted with chloroform. Chloroform should be used only in a well-ventilated area and inhalation of fumes should be avoided.

To increase the scientific value of tissue collections and voucher specimens, appropriate data (species, weight, standard measurements, reproductive condition, date, habitat, and specific locality of capture) should be recorded for each animal.

Necropsy and tissue collection.-Dissection scissors and forceps should be thoroughly cleaned and flamed over an alcohol burner before use or reuse. The ventral surface of the animal should be disinfected with alcohol and the peritoneal cavity opened, using blunt-end dissection scissors. The required organs should be removed with sterile, blunt-end forceps (use of scissors or toothed forceps is unnecessary and increases the risk of injury) and placed in labeled 2-ml cryovials. A single pair of forceps may be used to take all organs from each animal. Forceps should not touch pelage or working surfaces to avoid contamination. Scissors should not be used to take organs after they have been used to cut through the skin and peritoneum unless they are cleaned and flamed. Cryovials containing specimens should be tightly closed, wiped with disinfectant, and immediately placed on dry ice or in liquid nitrogen. The gloves and working surfaces should be disinfected between animals, and clean, flame-sterilized instruments (forceps and scissors) should be used for each animal. Used instruments should be placed into a disinfectant bath for the duration of the processing, or for $\geq 15 \mathrm{~min}$. Scissors and forceps from the disinfectant bath then should be carefully cleaned with a scrub brush under a fresh disinfectant solution and rinsed in clean water. Modern molecular techniques, such as polymerase chain reaction, are so sensitive that a minute amount of viral or host nucleic acid can result in false-positive results if carried over to subsequent samples. These clean instruments then should be flamed prior to reuse. If karyotypes are being prepared, long bones that are being flushed with a needle and syringe should be held with a hemostat to prevent accidental needlestick injury to the fingers or hand.
As a less desirable alternative to taking tissues from live-captured animals, samples may be obtained from animals freshly killed in snap traps. In this case, tissues should be taken within $2 \mathrm{~h}$ after capture or sooner if the ambient temperature is $>21^{\circ} \mathrm{C}\left(70^{\circ} \mathrm{F}\right)$. Fleas that may remain on the carcass can be killed with chloroform, and organs can be taken as previously described. A small amount of blood may be obtained from freshly kill-trapped rodents by opening the thoracic cavity and cutting the heart open with sterile blunt-end scissors. Blood then may be taken into a Pasteur pipette with rubber bulb or micropipetter with disposable tip and expelled into a labeled cryovial, taking care to avoid aerosol generation through bubbling or frothing. Alternatively, blood may be wicked into labeled, calibrated filter-paper strips, dried, and shipped to the processing laboratory in double plastic ziplock bags contained in a sturdy box. Nobuto filter strips for this purpose may be obtained from Microfiltration Systems (Dublin, CA). Directions provided with the strips should be followed.

Cleanup.-After all animals have been processed, all instruments and other items on the processing table should be wiped with disinfectant. Scissors and forceps from the disinfectant bath should be cleaned as previously described. Traps can be disinfected by placing each into a plastic container of disinfectant after the animal is removed. Bleach can be used but is not recommended for this purpose as it may corrode metal traps. Dirt and fecal material in traps can be removed with a long-handled brush while the trap is submerged in the container. After ca. 10 min in the disinfectant, the traps should be passed through one or two containers of clear rinse water. Heavy rubber gloves should be worn over latex gloves while handling traps to avoid tearing the latex gloves on sharp trap surfaces. All working surfaces, tables, chairs, and floor (if indoors) should be cleaned thoroughly with disinfectant. Disposable gowns, latex gloves, and contaminated trash should be placed into biohazard bags and disposed of in accordance with local requirements for infectious wastes. If processing was indoors, the area should be ventilated for $\geq 30 \mathrm{~min}$ before personnel should enter without respirators. Finally, hands should be thoroughly washed with soap and water after protective clothing is removed. If coveralls or gowns worn during processing are not disposa- 
ble, they should be laundered on site or immersed in liquid disinfectant until they can be washed. Potentially contaminated clothing should be laundered in hot water and detergent. Use rubber gloves when handling the soiled clothing and wash gloved hands in a disinfectant or soap and water, then remove gloves and wash hands with soap and water. Machine-dry laundry on a high setting or hang it to air dry in the sun.

Preparation of voucher specimens.-We recommend that what remains of each animal be prepared as a standard museum voucher specimen (Yates et al., in press). If this is not possible, carcasses should be double bagged and incinerated. If specimens are to be prepared as voucher specimens, they should be tagged (including all necessary data) and placed into a tight-sealing plastic container of $10 \%$ formalin for 7 days before handling. If animals are not opened to take tissue samples, they should be opened from lower abdomen to upper thorax to allow formalin to reach all tissues. To insure proper fixation, the volume of the carcasses should not exceed ca. $10 \%$ of the total volume of the container. After 1 week, carcasses should be placed in $70 \%$ alcohol for permanent storage. Foot tags should be made of $100 \%$ rag paper of ca. 110-lb. weight and should be attached to the right hind leg above the heel, using a strong, white cotton mercerized thread, size 10-12. Data on tags should be written with permanent black India ink or, if this is not available, a hard lead pencil (e.g., no. 4).

Standard museum skins and skeletons of freshly killed species that are known or suspected reservoirs of SNV or BCCV (Peromyscus and $S$. hispidus) must be prepared using all of the safety precautions previously discussed. Gloves, respirators, and protective clothing must be worn during specimen preparation, and biohazardous wastes should be incinerated or autoclaved. After removal, the skin and skeleton should be wetted thoroughly in $70 \%$ alcohol prior to stuffing with cotton or drying. These should then be quarantined and not handled without gloves until thoroughly dry. Although the survival of SNV outside of the host is not known, studies with related strains indicate that the virus may remain viable for as long as $48 \mathrm{~h}$ on dry surfaces (J. Huggins, pers. comm.).

\section{LABORATORY COLONIES}

Establishing or expanding colonies of wild rodents.-Special precautions should be taken when establishing laboratory colonies using wild rodents that are natural hosts of hantaviruses or other rodent-borne hemorrhagic fever viruses. Although experience is limited and detailed guidelines have not been established, these recommendations are based on current knowledge of hantavirus infection in host animals. They are to supplement guidelines promulgated by the animal care and use committee at each institution.

Laboratory transmission of hantaviruses to humans has resulted from exposure to rodent excreta, tissues (including cell lines), and suspended virus particles in the air at facilities where infected rodents were housed (Childs et al., 1995). Potential reservoir species (e.g., $R$. norvegicus, $S$. hispidus, and Peromyscus) should be quarantined and tested for hantavirus infection prior to housing them in an existing animal facility. Quarantine housing should be physically separated from other animal quarters with no air exchange with areas where humans work or other animals are housed. Individuals caring for the quarantined rodents should wear barrier clothing and respirators as previously specified.

Samples of whole blood should be taken from each rodent upon the initiation of quarantine and again after 30 days. If all samples are negative for antibody to hantaviruses, the animals can be housed in standard facilities, but in a separate room from standard laboratory strains of rodents to avoid transmission of other pathogens from the wild rodents. If any animals are seropositive, the entire quarantined colony should be euthanized and properly disposed. This precaution is necessary because some percentage of antibody-negative animals may carry virus (Childs et al., 1994).

Once the colony is established, periodic testing for hantaviruses (e.g., SNV and Seoul virus) should be done in compliance with institutional recommendations for certifying that laboratory colonies are free from zoonotic disease. This testing is important because interaction between feral 
and laboratory animals may introduce rodent-borne viruses capable of causing human disease into established colonies (e.g., lymphocytic choriomeningitis virus, Arenaviridae-Dykewicz, et al., 1992; Skinner et al., 1977).

Laboratory precautions.-Work with samples taken from potentially infected Peromyscus or S. hispidus should be conducted using biosafety-level-3 (BSL-3) practices (including controlled access, decontamination of wastes and laboratory clothing, "sharps" precautions, and baseline serum taken from technicians), although a BSL-2-containment facility (class I or II biosafety cabinets and autoclave available, use of laboratory coats, gloves, and face protection) can be used (Centers for Disease Control and Prevention, 1994). Work involving live, infected hosts or experimentally inoculated permissive hosts should be conducted at animal biosafetylevel-4 (ABSL-4; specially designed areas in which all personnel are required to wear positive-pressure suits ventilated with a life support system). See Centers for Disease Control and Prevention and National Institutes of Health (1993) for a complete explanation of biosafety levels and requirements.

\section{Existing Museum Collections}

Collections of research and teaching materials that contain samples of murid rodents generally are in the form of frozen tissues, museum study skins, osteological materials, or specimens preserved in formalin or alcohol. Traditional voucher specimens that have been preserved as dried skins plus osteological material or as fluidpreserved specimens for months or years do not pose a threat to humans from these viruses. Those specimens known to have been positive for a hantavirus, however, should be labeled as such directly on the tag or in the computer record associated with the specimen.

Tissue samples or entire specimens preserved under cryogenic conditions may har- bor live virus. In some situations, frozen whole carcasses may be used for hantavirus detection. When thawing and handling these materials, workers should use the precautions outlined under the Field Methods or Laboratory Precautions sections. Tissues known to have been taken from infected rodents should be labeled accordingly and notations entered into the appropriate database.

\section{CONCLUSIONS}

The outbreak of HPS in the United States during 1993 and the realization that the natural reservoir of SNV is one or several species of Peromyscus have had a profound impact on the science of mammalogy. Initial responses to these findings ranged from a lack of concern by some to cessation of all work with rodents by others. We believe a more balanced response is in order and that mammalogists should continue to conduct research and teaching activities with the addition of appropriate safety precautions as outlined. Continuing studies of hantaviruses and their host populations will provide additional data that will allow the improvement of current recommendations.

Risk of infection with hantaviruses appears to be relatively low among mammalogists, based on the low number of individuals who are seropositive (L. Armstrong et al., in litt.). Nevertheless, among the first 102 recognized cases of HPS in the United States, three were field biologists with a history of exposure to rodents ( $\mathrm{L}$. Armstrong et al., in litt.). Given the severity of HPS and the personal and institutional liability associated with potential infection of students or staff, all necessary precautions must be taken.

Students in field classes should refrain from handling mice in the genera Peromyscus and Sigmodon unless safety precautions described herein are followed. Students should avoid areas where these species are housed or studied, unless colonies are known to be hantavirus-free. When retrieving traps in the field, heavy leather or 
rubber gloves should be worn, and traps containing animals should be double bagged in plastic and returned to camp as previously described. Identification of species captured should be determined by a qualified mammalogist capable of specieslevel identifications and wearing adequate protective equipment as previously described. Again, traps that have contained animals should be decontaminated prior to being boxed or returned to the field.

Conducting fieldwork and handling wild animals always have involved a certain level of risk. Danger can be minimized by adequate training and knowledge of risks involved in each situation. Proper instruction for prevention of hantavirus infection is now part of mammalogical training as is avoidance of other zoonotic diseases, such as rabies, plague, and Lyme disease. It also should become part of our educational program for students in field biology.

The realization that a genus of viruses that contains at least some species that can be fatal when infecting humans and that apparently has evolved in close association with the family Muridae has been disconcerting to many. We believe this association also offers interesting possibilities and highlights another example of our limited understanding of biological diversity, even in relatively well-studied regions. It also reinforces the immense and largely untapped research potential represented by our museum collections of mammals.

\section{ACKNOWLEDGMENTS}

We thank B. Ellis, J. O'Connor, and H. Mathews for careful critiques of the manuscript. Use of trade names is for identification only and does not imply endorsement by the Public Health Service or the United States Department of Health and Human Services.

\section{Literature Cited}

Centers for Disease Control and Prevention. 1993. Hantavirus infection-southwestern United States: interim recommendations for risk reduction.
Morbidity and Mortality Weekly Report, 42(RR-11): $1-13$.

1994. Laboratory management of agents associated with hantavirus pulmonary syndrome: interim biosafety guidelines. Morbidity and Mortality Weekly Report, 43(RR-7):1-7.

Centers for Disease Control and PRevention and National Institutes of Health. 1993. Biosafety in microbiological and biomedical laboratories. Third ed., Health and Human Services Publication, United States Government Printing Office, Washington, D.C., (CDC)93-8395:1-177.

Childs, J. E., J. N. Mills, and G. E. Glass. 1995. Rodent-borne hemorrhagic fever viruses: a special risk for mammalogists? Journal of Mammalogy, 76: 664-680.

CHILDS, J. E., ET AL. 1994. Serologic and genetic identification of Peromyscus maniculatus as the primary rodent reservoir for a new hantavirus in the southwestern United States. Journal of Infectious Diseases, 169:1271-1280.

DYKEWICZ, C. A., ET AL. 1992. Lymphocytic choriomeningitis outbreak associated with nude mice in a research institute. Journal of the American Medical Association, 267:1349-1353.

DuCHIN, J. S., ET AL. 1994. Hantavirus pulmonary syndrome: a clinical description of 17 patients with a newly recognized disease. The New England Journal of Medicine, 330:949-955.

Glass, G. E., A. J. Watson, J. W. LeDuc, G. D. KeLEN, T. C. QuinN, AND J. E. ChILDS. 1993. Infection with a rat-borne hantavirus in United States residents is consistently associated with hypertensive renal disease. Journal of Infectious Diseases, 167:614 619.

HJELle, B., ET AL. 1994. Genetic identification of a novel hantavirus of the harvest mouse Reithrodontomys megalotis. Journal of Virology, 68:67516754.

LEDuC, J. 1987. Epidemiology of Hantaan and related viruses. Laboratory Animal Science, 37:413-418.

Lee, P.-W., H. L. Amyx, R. Yanagihara, D. C. GaJDUSEK, D. Goldgaber, AND C. J. GibBS, JR. 1985. Partial characterization of Prospect Hill virus isolated from meadow voles in the United States. Journal of Infectious Diseases, 152:826-829.

McKee, K. T., J. W. LeDuc, ANd C. J. Peters. 1991. Hantavirus. Pp. 615-632, in Textbook of human virology (R. B. Belshe, ed.). Mosby Year Book, St. Louis, Missouri, 1,064 pp.

RolLIN, P. E., ET AL. In press. Isolation of Black Creek Canal virus, a new hantavirus from Sigmodon hispidus in Florida. Journal of Medical Virology.

SKINNER, H. H., E. H. KNIGHT, AND R. GROVE. 1977. Murine lymphocytic choriomeningitis: the history of a natural cross-infection from wild to laboratory mice. Laboratory Animals, 11:219-222.

TSAI, T. 1987. Hemorrhagic fever with renal syndrome: mode of transmission to humans. Laboratory Animal Science, 37:428-430.

YATES, T. L., C. Jones, AND J. A. CoOK. In press. Preservation of voucher specimens. In Standardized methods of assessing and monitoring biodiversity in mammals (M. Foster and D. E. Wilson, eds.). Smithsonian Institution Press, Washington, D.C. 